\title{
Pharmaceutical Interventions on Hospital Discharge Prescriptions: Prospective Observational Study Highlighting Challenges for Community Pharmacists
}

\author{
Sophie Grandchamp ${ }^{1} \cdot$ Anne-Laure Blanc ${ }^{1} \cdot$ Marine Roussel $^{1} \cdot$ Damien Tagan $^{2} \cdot$ Annelore Sautebin $^{2}$. \\ Maria Dobrinas-Bonazzi ${ }^{1}$ (i) $\cdot$ Nicolas Widmer ${ }^{1,3}$
}

Accepted: 24 November 2021 / Published online: 31 December 2021

(c) The Author(s) 2021

\begin{abstract}
Background Transition between hospital and ambulatory care is a delicate step involving several healthcare professionals and presenting a considerable risk of drug-related problems.

Objective To investigate pharmaceutical interventions made on hospital discharge prescriptions by community pharmacists. Method This observational, prospective study took place in 14 community pharmacies around a Swiss acute care hospital. We recruited patients with discharge prescriptions (minimum three drugs) from the internal medicine ward of the hospital. The main outcome measures were: number and type of pharmaceutical interventions made by community pharmacists, time spent on discharge prescriptions, number of medication changes during the transition of care.

Results The study included 64 patients discharged from the hospital. Community pharmacists made a total of 439 interventions; a mean of $6.9 \pm 3.5$ (range 1-16) interventions per patient. All of the discharge prescriptions required pharmaceutical intervention, and 61 (95\%) necessitated a telephone call to the patients' hospital physician for clarifications. The most frequent interventions were: confirming voluntary omission of a drug (31.7\%), treatment substitution (20.5\%), dose adjustment (16.9\%), and substitution for reimbursement issues (8.8\%). Roughly half (52\%) of all discharge prescriptions required 10-20 min for pharmaceutical validation. The mean number of medication changes per patient was 16.4: 9.6 changes between hospital admission and discharge, 2.6 between hospital discharge and community pharmacy, and 4.2 between community pharmacy and a general practitioner's appointment.

Conclusion Hospital discharge prescriptions are complex and present a significant risk of medication errors. Community pharmacists play a key role in preventing and identifying drug-related problems.
\end{abstract}

Maria Dobrinas-Bonazzi and Nicolas Widmer contributed equally to the supervision of this work.

Maria Dobrinas-Bonazzi

maria.dobrinasbonazzi@phel.ch

1 Pharmacy of the Eastern Vaud Hospitals, Route du Vieux Séquoia 20, 1847 Rennaz, Switzerland

2 Riviera-Chablais Hospital, Vaud-Valais, Rennaz, Switzerland

3 Present Address: Institute of Pharmaceutical Sciences of Western Switzerland, University of Geneva, Geneva, Switzerland

\section{Key Points}

The number of pharmaceutical interventions made on discharge prescriptions by community pharmacists is significant, highlighting their essential role in the continuity of care after hospital discharge.

The validation of hospital discharge prescriptions is time-consuming and represents a constraint on community pharmacists and their patients.

The high number of medication changes during transitions of care creates a risk of medication errors and patient confusion, thus diminishing patient safety. 


\section{Introduction}

Patient safety has become a major preoccupation in recent years. Transitions between hospital and ambulatory care can be times of vulnerability for patients [1, 2], so ensuring continuity in patient care is essential. Insufficient transfers of information between hospitals and community pharmacies are associated with a high risk of drug-related problems (DRP) [3]. Nearly $49 \%$ of patients experienced at least one medical error related to the discontinuity of care between inpatient and outpatient settings, among which $42 \%$ were medication continuity errors [4]. Therefore, the optimization of patients' transfers between hospital and ambulatory care, as well as better communication between healthcare professionals, have all shown good clinical outcomes [5].

All healthcare professionals can play a role in facilitating transitions from secondary to primary care; community pharmacists can offer accessibility, expertise in drug therapeutics, face-to-face contact, and skills in managing DRPs and therapeutic adherence [2]. One of a community pharmacist's main activities is validating medical prescriptions, which involves detecting any potential problems in the prescription and suggesting potential changes to drug therapies, both defined as pharmaceutical interventions [6]. Community pharmacists can therefore play an active role in preventing and solving DRPs [3, 7].

Hospital discharge prescriptions present a particularly high risk of DRPs [8]. The number of prescribed drugs itself can represent a risk factor. However, it is the discrepancies between the pre-hospitalization and post-discharge treatments that are of major concern. Only $10 \%$ of patients are discharged with the same medication as at hospital admission [2]. Different problems requiring pharmaceutical interventions have been identified, such as drug omissions, medication discontinued unintentionally, inappropriate medication schedules, adverse drug events, drug-drug interactions, and inappropriate dosage $[3,7]$. Furthermore, because community pharmacists are often the final link in the transition of care, they may represent the last opportunity to identify DRPs before new drug regimens are delivered to ambulatory patients [8, 9].

In Swiss ambulatory care, a patient's medication is usually prescribed by the family general practitioner (GP) and dispensed by the community pharmacy. After a hospital stay, patients are provided with a discharge prescription, which allows discharge medicines to be filled at the community pharmacy. In parallel, a discharge summary along with the prescription are sent to the patient's GP, who also receives a complete report some weeks later, and meets the patient for establishing the final therapeutic plan. However, the discharge summary is usually not available for the community pharmacist and for the patient. Therefore, community pharmacists often lack information when validating the discharge prescription and reconciling it with a patient's medication history that is available in their pharmacy software (drug discontinuation, reason for drug substitution, dosage changes, etc.). They attempt to complete missing information by calling the patients' hospital physicians for clarifications. Further on, communication with the GP might be established for the long-term follow-up of patients, but this is not systematically done at hospital discharge.

In a recent study, Swiss community pharmacists expressed the need for health-related and care-related information, which is still inaccessible, together with therapyrelated information, which can be improved. They called for enhanced collaboration to support patient safety, mainly through information transfer, which was hoped to be concise and well structured to enable quick and easy reading [10].

\section{Aim of the Study}

The present exploratory study aimed to investigate the pharmaceutical interventions made by community pharmacists on the hospital discharge prescriptions from an internal medicine department.

\section{Method}

\subsection{Setting and Participants}

This observational, prospective study took place in community pharmacies around a Swiss regional hospital (the Riviera-Chablais Hospital, Vaud-Valais). Their staff recruited consecutively, from October 2015 to December 2015, all patients who fulfilled the following inclusion criteria: discharged from the hospital's internal medicine ward; taking more than three drugs for chronic complaints; proficient in French; aged 18 years or more; being monitored by one of the participating community pharmacies; a hospital stay that lasted at least 3 days; capable of discernment; and providing informed oral consent. All informed oral consents were documented by the participating community pharmacists. The Cantonal Ethics Commitee Vaud, which approved the study, ruled that this oral agreement was acceptable.

\subsection{Outcomes and Variables}

Participating community pharmacists were asked to collect data about all the interventions they made and the time required to deal with the hospital discharge prescriptions. A 
Table 1 Data collection questionnaire for interventions made by community pharmacists

\begin{tabular}{ll}
\hline Problems identified & Interventions \\
\hline Pharmaceutical problems & Confirming voluntary omission of a drug taken before \\
hospitalization with the patient's hospital physician \\
Dose adjustment according to medical history \\
Treatment substitution (active ingredient) \\
Treatment substitution (brand name) \\
Initiation of a drug omitted from the discharge prescription \\
Drug-drug interactions \\
Cancelling duplicate therapy \\
Optimization of treatment duration or schedule \\
Over-dosage \\
Under-dosage \\
Side effects \\
Contraindications \\
Drug addiction \\
Optimization of drug quantity \\
Optimization of galenic formulation \\
Clarification of the medical record \\
Medication substitution because of reimbursement issues \\
Delayed narcotic drug prescription \\
Dractical issues & Drug unavailable in the pharmacy \\
Logistical constraints &
\end{tabular}

data collection questionnaire for the study was adapted from Maes et al. [11] and is presented in Table 1.

One of the pharmacists responsible for the study made a weekly visit to the community pharmacies and collected the following information on each participating patient: documentation of the informed oral consent, the data collection questionnaire completed by community pharmacists; the medication history for the 3 months before hospitalization, extracted using the pharmacy software; and the treatments delivered to the patient in the month following hospital discharge, according to the new prescription issued by the patient's GP.

Demographic data (age, sex) and clinical data (diagnoses, comorbidities) were collected retrospectively from patients' electronic medical records (Soarian ${ }^{\circledR}$ version 4.00 SP08, Cerner Health Services, Kansas City, MO, USA) corresponding to their hospital stay. Diagnoses and comorbidities were subsequently classified into broader categories. Drugs were recorded by trade name and classified according to the anatomical therapeutic chemical (ATC) classification system [12].

The outcomes analyzed were the number and type of interventions made by community pharmacists, the therapeutic classes involved in those interventions, other pharmaceutical suggestions made by the pharmacists, and the time spent validating hospital discharge prescriptions (including for possible telephone calls to patients' hospital physicians for clarifications).
The data collection questionnaire listed several possible types of interventions covering pharmaceutical problems, practical issues, and logistical constraints (Table 1).

The community pharmacists could suggest other ways to improve patients' treatment (defined as a pharmaceutical suggestions) such as a written summary of discharge medication, therapeutic drug monitoring, support for therapeutic adherence, a polymedication review, and use of a pill-box. A polymedication review is a community pharmacy service, that was reimbursed under Swiss health insurance laws at the time of study. It includes teaching patients how to use their prescribed drugs, counseling on potential side effects and drug-drug interactions, and evaluating therapeutic adherence [13].

The number of medication changes at different steps in the transitions was also noted, enabling a comparison of medication histories between hospital admission and discharge, hospital discharge and community pharmacy care, and community pharmacy care and appointments with GPs 30 days after discharge. The types of medication changes possible at each step included initiating a new medication, discontinuing an existing medication, reinitiating previously discontinued medication, drug substitution, dose changes, changes in drug regimen and treatment duration, and changes in galenic formulation. 


\subsection{Statistical Analysis}

Descriptive statistics (means, medians, proportions, standard deviations, confidence intervals, and their graphic representations) were calculated using Excel $^{\circledR}$ (version 2010, Microsoft Corp, Redmond, WA, USA).

\section{Results}

\subsection{Patients' Characteristics}

Fourteen of the 37 community pharmacies invited to participate in the study did so, and they recruited 64 patients over a 3-month period. Patients' characteristics, diagnoses, and comorbidities are presented in Table 2.

\subsection{Community Pharmacists' Validation of Hospital Discharge Prescriptions}

Community pharmacists made 439 interventions on the 64 hospital discharge prescriptions included in this study. The mean number of pharmaceutical interventions per patient was 6.9 ( $\mathrm{SD} \pm 3.5$; range $1-16)$, and every discharge prescription required at least one intervention. The different types of interventions are presented in Table 3.

Community pharmacists spent significant amounts of time dealing with discharge prescriptions: roughly half $(52 \%)$ of patient discharge prescriptions required between 10 and $20 \mathrm{~min}$, $14 \%$ required less than $10 \mathrm{~min}, 6 \%$ required more than $40 \mathrm{~min}$, and the remaining $28 \%$ required between 20 and $40 \mathrm{~min}$.

Of the 64 prescriptions, 61 (95\%) necessitated telephone calls to patients' hospital physicians to clarify medication changes. This corresponds to a total number of $303(69 \%)$ interventions and a mean number of $4.8(\mathrm{SD} \pm 3.1$; range 0-14) interventions per patient requiring a telephone call.

Community pharmacists made 37 pharmaceutical suggestions on the 64 prescriptions included in the study. Among them, using a pill-box was recommended to $19(51 \%)$ patients, a written summary of the discharge medication was provided to seven $(19 \%)$ patients, therapeutic drug monitoring was suggested to five (14\%) patients, and support for therapeutic adherence together with a polymedication review were offered to three $(8 \%)$ patients.

The community pharmacists made pharmaceutical interventions on discharge prescriptions involving 47 different therapeutic drug classes. Analgesics were the most frequently involved drugs (17\%) with the main interventions being reimbursement issues and dose adjustment according to medication history. These were followed by mineral supplements (10\%; main interventions: treatment substitution and dose adjustment according to medication history), psychotropic drugs (7\%; main interventions: treatment substitution and confirming voluntary omission), drugs for acid-related disorders (6\%; main interventions: treatment substitution and dose adjustment according to medication

Table 2 Patients' characteristics

\begin{tabular}{lll}
\hline Characteristics & Study group $(n=64)$ & \\
\hline Age, mean \pm SD (range), years & $78 \pm 12(44-98)$ & $(\%)$ \\
Sex & $n$ & $40.6 \%$ \\
Male & 26 & $59.4 \%$ \\
Female & 38 & \\
Number of drugs on discharge prescription, mean \pm SD (range) & $10 \pm 4(3-19)$ & $(\%)$ \\
Length of hospital stay, mean \pm SD (range), days & $18 \pm 15(2-57)$ & $19 \%$ \\
Diagnoses and comorbidities & $n$ & $28 \%$ \\
Myocardial infarction & 12 & $8 \%$ \\
Congestive heart failure & 18 & $13 \%$ \\
Peripheral vascular disease & 5 & $9 \%$ \\
Cerebrovascular disease & 8 & $23 \%$ \\
Dementia & 6 & $6 \%$ \\
Chronic pulmonary disease & 15 & $11 \%$ \\
Rheumatological disease & 4 & $25 \%$ \\
Peptic ulcer disease & 7 & $3 \%$ \\
Diabetes without chronic complications & 16 & $17 \%$ \\
Diabetes with chronic complications & 2 & $2 \%$ \\
Renal disease & 11 & $3 \%$ \\
Any malignancy, including leukemia or lymphoma & 1 & $2 \%$ \\
Moderate or severe liver disease & 2 & \\
Metastatic solid tumor & 1 & \\
\hline
\end{tabular}


Table 3 Pharmaceutical interventions by community pharmacists

\begin{tabular}{|c|c|c|c|}
\hline Pharmaceutical intervention & Number & Percentage & Number/patient \\
\hline Confirming voluntary omission of a drug taken before hospitalization & 139 & $31.7 \%$ & 2.2 \\
\hline Dose adjustment according to medical history & 74 & $16.9 \%$ & 1.2 \\
\hline Treatment substitution (active ingredient) & 49 & $11.2 \%$ & 0.8 \\
\hline Treatment substitution (brand name) & 41 & $9.3 \%$ & 0.6 \\
\hline Medication substitution because of a reimbursement issue & 39 & $8.9 \%$ & 0.6 \\
\hline Optimization of galenic formulation & 29 & $6.6 \%$ & 0.5 \\
\hline Confirming initiation of a drug omitted from the discharge prescription & 28 & $6.4 \%$ & 0.4 \\
\hline Unavailable drug & 14 & $3.2 \%$ & 0.2 \\
\hline Delayed narcotic drug prescription & 7 & $1.6 \%$ & 0.1 \\
\hline Drug-drug interactions & 5 & $1.1 \%$ & 0.1 \\
\hline Optimization of drug quantity & 5 & $1.1 \%$ & 0.1 \\
\hline Over-dosage & 2 & $0.5 \%$ & \\
\hline Clarification of medical record & 2 & $0.5 \%$ & \\
\hline Under-dosage & 1 & $0.2 \%$ & \\
\hline Drug dependency & 1 & $0.2 \%$ & \\
\hline Duplicate therapy & 1 & $0.2 \%$ & \\
\hline Optimization of treatment duration & 1 & $0.2 \%$ & \\
\hline Drug unavailable on the market & 1 & $0.2 \%$ & \\
\hline
\end{tabular}

history), and agents acting on the renin-angiotensin system (6\%; main interventions: confirming voluntary omission and treatment substitution).

\subsection{Medication Changes During Transition of Care}

The mean number of medication changes per patient over all the different steps in the transition of care was 16.4 (SD \pm 7.3 ; range 2-38): 9.6 (SD \pm 4.5 ; range $2-23$ ) changes between hospital admission and discharge, the most frequent being the initiation and interruption of treatments; $2.6(\mathrm{SD} \pm 1.9$; range $0-9$ ) changes between hospital discharge and community pharmacy care, the most frequent being treatment substitution (either brand name or active ingredient); and 4.2 (SD \pm 2.7 ; range $0-12$ ) changes between community pharmacy care and an appointment with a GP 30 days after hospital discharge, the most frequent being interruption of treatment and initiation of a drug omitted from the discharge prescription. It is worth noting that one-third of patients $(n=21)$ did not visit their GP during the month following hospital discharge.

\section{Discussion}

\subsection{Community Pharmacists' Validation of Hospital Discharge Prescriptions}

In the present study, community pharmacists made 439 interventions on 64 hospital discharge prescriptions-a mean of 6.9 interventions per prescription.
Other studies have also evaluated community pharmacists' interventions on hospital discharge prescriptions. However, depending on study designs and definitions used, results are presented as DRPs encountered, interventions made, or both [3, 6-9, 14, 15]. For clarity, in the present study, pharmaceutical interventions refers to any interventions made by community pharmacists to resolve any problems identified, whether pharmaceutical problems, practical issues, or logistical constraints.

Every discharge prescription in the present study required at least one pharmaceutical intervention, and we reported more pharmaceutical interventions than other studies focusing on primary-care prescriptions managed by community pharmacists. For instance, the overall rate of prescriptionrelated problems was of $0.37 \%$ in a French study [6] and of $1.5 \%$ in a Spanish study [9]. Another study focusing on the geriatric population in community pharmacies identified DRPs in $37.3 \%$ of the patients included in the study, with an average of 1.37 DRPs per patient [16]. Other communitybased studies assessing medical prescriptions in elderly patients in the Netherlands [14], in China [17], and in Belgium [18] reported results closer to our study: $3.9,4.8$, and 3 DRPs per patient, respectively. These differences may be explained partly by the fact that these studies included all prescriptions analyzed by community pharmacists, not only hospital discharge prescriptions.

Differences were still observed when results were compared with those from studies focusing only on hospital discharge prescriptions. In one study, $25 \%$ of hospital discharge prescriptions required interventions from community 
pharmacists, with an average of 1.2 DRPs per patient [3]. Two other studies identified DRPs in 63.7 and $38 \%$ of patients with hospital discharge prescriptions, respectively. However, other studies reported similar results to ours. Thus, community pharmacists identified DRPs in $93.3 \%$ of patients discharged from the cardiology unit of an Australian hospital, with a mean of 5.6 DRPs per patient [19]. Another French study reported at least one DRP for nearly all patients (95.9\%), with a mean of 2.9 DRPs per patient [15]. A recent study built on the collaboration between hospital and community pharmacists identified an average of 4.9 DRPs per patient, the most common DRPs being "need for education or information" and "compliance issues" [20]. The higher number of pharmaceutical interventions reported in our study may be explained by our broader definition of intervention, as described above.

Two-thirds of the present study's interventions necessitated a telephone call to the patient's hospital physician to confirm medication changes. In a study in New Zealand, only $8 \%$ of interventions necessitated a telephone call-a much lower rate, but perhaps explainable by the types of interventions made [8]. In the New Zealand study, the most frequent pharmaceutical intervention concerned medication availability, which does not necessitate calling the patient's physician. On the contrary, four of our study's main pharmaceutical interventions (confirming voluntary omission of a drug, dose adjustment, treatment substitution, and initiation of an omitted drug) necessitate calling the patient's physician. Similarly, a second New Zealand study found that $19 \%$ of interventions necessitated calling the patient's physician [3].

Approximately half of our study's interventions involved voluntary omissions of drugs taken before hospitalization or dose adjustments according to medical histories. Other reasons for pharmaceutical interventions were incomplete medication histories or discrepancies in medication reconciliation, as previously described [21-23], which could be reduced by better pharmaceutical anamnesis. Indeed, clinical pharmacists can be involved in this, as shown by a study in which anamneses performed by clinical pharmacists improved medication reconciliation processes in elderly patients [24].

In the present study, interventions involving treatment substitution, reimbursement issues, or optimization of galenic formulations were often caused by the need to align with the hospital's formulary during the stay. For instance, paracetamol (acetaminophen; Panadol ${ }^{\circledR}$ ), an analgesic in the hospital's formulary, is not reimbursed in community care as an original formulation. To ensure reimbursement, therefore, community pharmacists must replace it with another paracetamol-containing medication. The same situation applies for galenic formulations: the hospital's formulary only contains one dosage, but community pharmacists often suggest another dosage or formulation to optimize a patient's treatment. In case of therapeutic substitutions, community pharmacists needed to call the patient's hospital physician to clarify whether the substitution was made in order to align with the hospital's formulary during the stay (in this case allowing a return to the previous medication), or the substitution was linked to a medical reason, which required continuation of the hospital medication.

Ideally, medication reconciliation should be done before hospital discharge, and any necessary changes should be included on the discharge prescription, accompanied by the explanations for these changes for the community pharmacists.

Another reason for treatment substitution is to propose less costly medication, especially when patients accept brand-to-generic substitutions. Money-saving treatment substitutions are encouraged through the performance-based remuneration of community pharmacists by health insurance companies [13].

The therapeutic classes most affected by pharmaceutical interventions were analgesics, mineral supplements, psychotropic drugs, drugs for acid-related disorders, and agents acting on the renin-angiotensin system. Interventions involving analgesic drugs were mainly treatment substitutions for reimbursement issues, as mentioned above for paracetamol. Mineral supplements were mainly affected by dose adjustments and treatment substitutions, especially when medication taken before hospitalization was reintroduced; dose adjustments were due to differences in dosages of calcium and cholecalciferol, depending on the brand name.

The most frequent interventions involving psychotropic drugs were treatment substitutions, confirming deliberate drug omissions, or initiating omitted drugs. In most cases, upon hospital admission, patients had not mentioned that they were taking psychotropic drugs (e.g., benzodiazepines for sleep disorders), so they were omitted during hospital stay. Later on, these circumstances were confirmed when community pharmacists called patients' hospital physicians. This specific issue was explored by our team in a previous study focusing on the prescription of hypnotic drugs during hospital stays [25]. Drugs for acid-related disorders were involved in treatment substitutions for the drug taken before hospital stay, dose adjustments, and confirmations of deliberate drug omissions if the indication was unclear. Cardiac medications are often the subject of pharmaceutical interventions [7]. In the present study, agents acting on the renin-angiotensin system were sometimes omitted at hospital admission and were frequently replaced by another active ingredient available on the formulary.

In this study, more than half (52\%) of hospital discharge prescriptions took between 10 and $20 \mathrm{~min}$ to be validated by community pharmacists, and $28 \%$ took between 20 and $40 \mathrm{~min}$. Our results are similar to results of another study, 
in which community pharmacists took an average of $45 \mathrm{~min}$ to analyze hospital discharge prescriptions, including a mean time of 11 min counseling patients [21]. These results show that the validation of hospital discharge prescriptions is time-consuming, representing a potential limitation on other community pharmacy tasks, but also on patients (who usually wish to get home as soon as possible after hospital discharge).

Using a pill-box was the most frequent suggestion made by community pharmacists. In Switzerland, community pharmacists can suggest the use of a pill-box, which will be reimbursed after obtaining a prescription from the patient's GP. Alternatively, a pill-box could be previously provided for free for the 3 months following a polymedication review [13].

\subsection{Medication Changes During Transitions of Care}

The mean number of medication changes per patient in this study was 16.4. The highest number of medication changes took place between hospital admission and hospital discharge, and the most frequent were the initiation or interruption of treatments. These results are similar to previously published data: $28 \%$ of prior medications were stopped upon hospital admission [26]. Another study showed that during hospitalization, $36 \%$ of drugs were interrupted, $46 \%$ were newly prescribed, $7 \%$ involved dose changes, and $6 \%$ involved treatment substitutions [27]. Our results were similar, with $26,40,12$, and $10 \%$ of drugs interrupted, newly prescribed, involving dose changes, and substituted, respectively.

Furthermore, other changes occur when patients visit their GPs: they often return patients to their pre-hospitalization treatments. Indeed, in the present study, the most frequent changes at this step were treatment interruptions and substitutions. The study focusing on the number of medication changes between hospital discharge and a first visit to the GP found that the GP interrupted $13 \%$ of drugs and changed $21 \%$ [26], compared to $38 \%$ of drugs discontinued and $20 \%$ substituted in our study. However, one-third of our study's patients did not see their GP in the month following discharge. This could affect quality of care because, ideally, GPs should validate changes made during hospital stays and prescribe the patients' latest treatments.

Although medication changes are to be expected after a hospitalization, they can create misunderstandings with patients, communication of information may be incomplete during transition steps, medication safety may decrease [28, 29], and related costs may increase [29].

Moreover, discharge can often be a time of confusion for patients, and most of them are unable to name their medication, diagnoses, drugs' roles, and their main side effects. Appropriate pharmaceutical interventions to improve hospital discharge prescriptions could also improve patients' awareness, understanding, and adherence to treatment [30].

Most of the pharmaceutical interventions made in our study emphasize the difficulties community pharmacists face when dealing with hospital discharge prescription, some of which have also been described in other publications. A study analyzing the barriers and facilitators in medication reconciliation for recently discharged patients showed that community pharmacists felt reconciliation was a "standard of care" for pharmacists, but they found the process to be difficult and time-consuming. Several barriers to medication reconciliation were identified: not having access to the patient's medical record (lab tests, indications, medication histories), difficulties in reaching the hospital physician to clarify the prescription, lack of sufficient pharmacy staff, lack of reimbursement for this activity. Among possible facilitators, pharmacists identified the "transitional care teams" who could help by providing the pharmacist with alerts when patients are being discharged, receiving "stop orders" for drug discontinuations, and assuring information transfer through electronic modes [31].

In another study, community pharmacists faced several issues following a patient's hospital discharge, mainly related to prescription quality or to logistic concerns. These risks were higher in patients who were discharged without being given any information about their medication. Another challenge was the fact that many patients did not show up in a timely manner with their prescription at community pharmacies, possibly leading to discontinuation of treatment and hence to potential clinical complications. In order for community pharmacists to be able to successfully ensure continuous patient care, information provided by hospital healthcare professionals needs to be improved [32].

Ensuring that patients and community pharmacists receive better information at every step in the transition of care could reduce the number of pharmaceutical interventions required on hospital discharge prescriptions, as our research group recently demonstrated [33]. Other publications highlighted the need of interventions during hospital stay intended to improve information transfer (such as a discharge medication report), which could reduce medication errors after hospital discharge [21, 34, 35].

\subsection{Study Strengths and Limitations}

This study had several limitations. Firstly, the number of community pharmacies, and therefore the number of patients, included was rather small. Larger multicentric studies are needed in order to generalize these results. Secondly, we did not consider the potential for differences in pharmacy 
practices among the 14 local community pharmacies who participated. However, the standardized data collection related to pharmaceutical interventions and medication histories was performed by a single investigator working with all the community pharmacies involved in the study. Thirdly, medication histories are not always complete in community pharmacies, especially with regard to over-the-counter medications (laxatives, NSAIDs, etc.). This may have underestimated the number of pharmaceutical interventions. Lastly, it was not possible in this study to demonstrate the severity or impact of each pharmaceutical interventions on patients' clinical outcomes (neutral, worsen clinical outcome, risk of toxicity, etc.). This constitutes a further research perspective. However, several strengths of the study can be mentioned: the study was built on robust collaboration between the hospital and community pharmacists, it was a prospective study using a systematic methodology to identify the challenges related to hospital discharge prescriptions, and it took place in a real-life setting.

\section{Conclusion}

The present study emphasized the complexity and challenges related to hospital discharge prescriptions. Community pharmacists play a key role in identifying and preventing DRPs, but the time required for pharmaceutical validation might be a constraint upon pharmacists and patients. Medication reconciliation at hospital admission and more effective communication of medication changes at discharge could facilitate community pharmacists' work. Improved communication between different healthcare professionals and their patients can increase patient safety and ensure continuity of care.

Acknowledgements The authors are thankful to the community pharmacies around the study hospital for their valuable work and collaboration, and to all the patients who participated in this study. Finally, D. Hart is thanked for proofreading the English.

\section{Declarations}

Funding No funding was received for this study.

Conflict of interest The authors declare that they have no competing interests.

Availability of data and material Data are available on request from the authors.

Ethics approval This study was approved by the Human Research Ethics Committee of the Canton Vaud (approval ID: 358/15).

Consent Informed oral consent was obtained from each patient included in the study.
Author contributions ALB, DT, AS, and NW contributed to the conception of the study. All the authors contributed to the study design. SG and MR contributed to data collection and analysis. SG, MR, ALB, $\mathrm{MDB}$, and NW contributed to the analysis of publications, data analysis and their interpretation. SG and MDB drafted the article, and it was reviewed by the rest of the authors. Finally, all authors read and approved the final manuscript.

Open Access This article is licensed under a Creative Commons Attribution-NonCommercial 4.0 International License, which permits any non-commercial use, sharing, adaptation, distribution and reproduction in any medium or format, as long as you give appropriate credit to the original author(s) and the source, provide a link to the Creative Commons licence, and indicate if changes were made. The images or other third party material in this article are included in the article's Creative Commons licence, unless indicated otherwise in a credit line to the material. If material is not included in the article's Creative Commons licence and your intended use is not permitted by statutory regulation or exceeds the permitted use, you will need to obtain permission directly from the copyright holder. To view a copy of this licence, visit http://creativecommons.org/licenses/by-nc/4.0/.

\section{References}

1. Riordan CO, Delaney T, Grimes T. Exploring discharge prescribing errors and their propagation post-discharge: an observational study. Int J Clin Pharm. 2016;38(5):1172-81. https://doi.org/10. 1007/s11096-016-0349-7.

2. Nazar H, Nazar Z, Portlock J, Todd A, Slight SP. A systematic review of the role of community pharmacies in improving the transition from secondary to primary care. Br J Clin Pharmacol. 2015;80(5):936-48. https://doi.org/10.1111/bcp.12718.

3. Braund R, Coulter CV, Bodington AJ, Giles LM, Greig AM, Heaslip LJ, et al. Drug related problems identified by community pharmacists on hospital discharge prescriptions in New Zealand. Int J Clin Pharm. 2014;36(3):498-502. https://doi.org/10.1007/ s11096-014-9935-8.

4. Moore C, Wisnivesky J, Williams S, McGinn T. Medical errors related to discontinuity of care from an inpatient to an outpatient setting. J Gen Intern Med. 2003;18(8):646-51. https://doi.org/10. 1046/j.1525-1497.2003.20722.x.

5. Dvorak SR, McCoy RA, Voss GD. Continuity of care from acute to ambulatory care setting. Am J Health Syst Pharm. 1998;55(23):2500-4. https://doi.org/10.1093/ajhp/55.23.2500.

6. Théo L. Analyse des interventions pharmaceutiques réalisées lors de la validation des prescriptions médicales, au sein d'une officine de pharmacie. J Pharm Clin. 2011;30(3):155-8. https://doi.org/10. 1684/jpc.2011.0178.

7. Paulino EI, Bouvy ML, Gastelurrutia MA, Guerreiro M, Buurma H, Group E-SRCPR. Drug related problems identified by European community pharmacists in patients discharged from hospital. Pharm World Sci. 2004;26(6):353-60.

8. Maxwell K, Harrison J, Scahill S, Braund R. Identifying drugrelated problems during transition between secondary and primary care in New Zealand. Int J Pharm Pract. 2013;21(5):333-6. https:// doi.org/10.1111/ijpp.12013.

9. Martínez Sánchez A, Campos RM. Detection of prescribing related problems at the community pharmacy. Int J Clin Pharm. 2011;33(1):66-9. https://doi.org/10.1007/s11096-010-9459-9.

10. Bruhwiler LD, Hersberger KE, Lutters M. Hospital discharge: What are the problems, information needs and objectives of community pharmacists? A mixed method approach. Pharm Pract 
(Granada). 2017;15(3):1046. https://doi.org/10.18549/Pharm Pract.2017.03.1046.

11. Maes KA, Tremp RM, Hersberger KE, Lampert ML. Demonstrating the clinical pharmacist's activity: validation of an intervention oriented classification system. Int J Clin Pharm. 2015;37(6):116271. https://doi.org/10.1007/s11096-015-0179-z.

12. WHO Collaborating Centre for Drug Statistics Methodology Anatomical Therapeutic Chemical (ATC) classification system. 2020. http://www.whocc.no/copyright_disclaimer/.

13. Curafutara, PharmaSuisse, SantéSuisse. Convention relative à la structure tarifaire RBP IV/1. 2016. https://www.tarifsuisse.ch/filea dmin/sas_content/Convention_relative_a_la_structure_tarifaire_ RBP_IV.pdf.

14. Vinks TH, de Koning FH, de Lange TM, Egberts TC. Identification of potential drug-related problems in the elderly: the role of the community pharmacist. Pharm World Sci. 2006;28(1):33-8. https://doi.org/10.1007/s11096-005-4213-4.

15. Ahmad A, Mast MR, Nijpels G, Elders PJ, Dekker JM, Hugtenburg JG. Identification of drug-related problems of elderly patients discharged from hospital. Patient Prefer Adher. 2014;8:155-65. https://doi.org/10.2147/PPA.S48357.

16. Rhalimi M, Rauss A, Housieaux E. Drug-related problems identified during geriatric medication review in the community pharmacy. Int J Clin Pharm. 2018;40(1):109-18. https://doi.org/10. 1007/s11096-017-0571-y.

17. Yang J, Meng L, Liu Y, Lv L, Sun S, Long R, et al. Drug-related problems among community-dwelling older adults in mainland China. Int J Clin Pharm. 2018;40(2):368-75. https://doi.org/10. 1007/s11096-017-0587-3.

18. Wuyts J, Maesschalck J, De Wulf I, Lelubre M, Foubert K, De Vriese C, et al. Studying the impact of a medication use evaluation by the community pharmacist (Simenon): drug-related problems and associated variables. Res Social Adm Pharm. 2020;16(8):1100-10. https://doi.org/10.1016/j.sapharm.2019. 11.008 .

19. Ellitt GR, Engblom E, Aslani P, Westerlund T, Chen TF. Drug related problems after discharge from an Australian teaching hospital. Pharm World Sci. 2010;32(5):622-30. https://doi.org/10. 1007/s11096-010-9406-9.

20. Ensing HT, Koster ES, Dubero DJ, van Dooren AA, Bouvy ML. Collaboration between hospital and community pharmacists to address drug-related problems: the HomeCoMe-program. Res Soc Adm Pharm. 2019;15(3):267-78. https://doi.org/10.1016/j.sapha rm.2018.05.001.

21. Geurts MM, van der Flier M, de Vries-Bots AM, Brink-van der Wal TI, de Gier JJ. Medication reconciliation to solve discrepancies in discharge documents after discharge from the hospital. Int J Clin Pharm. 2013;35(4):600-7. https://doi.org/10.1007/ s11096-013-9776-x.

22. Wong JD, Bajcar JM, Wong GG, Alibhai SM, Huh JH, Cesta A, et al. Medication reconciliation at hospital discharge: evaluating discrepancies. Ann Pharmacother. 2008;42(10):1373-9. https:// doi.org/10.1345/aph.1L190.

23. Cornish PL, Knowles SR, Marchesano R, Tam V, Shadowitz S, Juurlink DN, et al. Unintended medication discrepancies at the time of hospital admission. Arch Intern Med. 2005;165(4):424-9. https://doi.org/10.1001/archinte.165.4.424.

24. Steurbaut S, Leemans L, Leysen T, De Baere E, Cornu P, Mets $\mathrm{T}$, et al. Medication history reconciliation by clinical pharmacists in elderly inpatients admitted from home or a nursing home. Ann Pharmacother. 2010;44(10):1596-603. https://doi.org/10.1345/ aph.1P192.

25. Schumacher L, Dobrinas M, Tagan D, Sautebin A, Blanc AL, Widmer N. Prescription of sedative drugs during hospital stay: a Swiss prospective study. Drugs Real World Outcomes. 2017;4(4):225-34. https://doi.org/10.1007/s40801-017-0117-6.

26. Himmel W, Tabache M, Kochen MM. What happens to long-term medication when general practice patients are referred to hospital? Eur J Clin Pharmacol. 1996;50(4):253-7. https://doi.org/10.1007/ s002280050103.

27. Himmel W, Kochen MM, Sorns U, Hummers-Pradier E. Drug changes at the interface between primary and secondary care. Int $\mathrm{J}$ Clin Pharmacol Ther. 2004;42(2):103-9. https://doi.org/10.5414/ cpp42103.

28. Frydenberg K, Brekke M. Poor communication on patients' medication across health care levels leads to potentially harmful medication errors. Scand J Prim Health Care. 2012;30(4):234-40. https://doi.org/10.3109/02813432.2012.712021.

29. Kripalani S, Henderson LE, Jacobson TA, Vaccarino V. Medication use among inner-city patients after hospital discharge: patient-reported barriers and solutions. Mayo Clin Proc. 2008;83(5):529-35. https://doi.org/10.4065/83.5.529.

30. Makaryus AN, Friedman EA. Patients' understanding of their treatment plans and diagnosis at discharge. Mayo Clin Proc. 2005;80(8):991-4. https://doi.org/10.4065/80.8.991.

31. Kennelty KA, Chewning B, Wise M, Kind A, Roberts T, Kreling D. Barriers and facilitators of medication reconciliation processes for recently discharged patients from community pharmacists' perspectives. Res Soc Adm Pharm. 2015;11(4):517-30. https:// doi.org/10.1016/j.sapharm.2014.10.008.

32. Michel B, Hemery M, Rybarczyk-Vigouret MC, Wehrle P, Beck M. Drug-dispensing problems community pharmacists face when patients are discharged from hospitals: a study about 537 prescriptions in Alsace. Int J Quality Health Care. 2016;28(6):779-84. https://doi.org/10.1093/intqhe/mzw111.

33. Neeman M, Dobrinas M, Maurer S, Tagan D, Sautebin A, Blanc AL, et al. Transition of care: a set of pharmaceutical interventions improves hospital discharge prescriptions from an internal medicine ward. Eur J Intern Med. 2017;38:30-7. https://doi.org/ 10.1016/j.ejim.2016.11.004.

34. Midlov P, Holmdahl L, Eriksson T, Bergkvist A, Ljungberg B, Widner $\mathrm{H}$, et al. Medication report reduces number of medication errors when elderly patients are discharged from hospital. Pharm World Sci. 2008;30(1):92-8. https://doi.org/10.1007/ s11096-007-9149-4.

35. Cua YM, Kripalani S. Medication use in the transition from hospital to home. Ann Acad Med Singap. 2008;37(2):136-46. 